\title{
Genome Sequence of Rhizoctonia solani Anastomosis Group 4 Strain Rhs4ca, a Widespread Pathomycete in Field Crops
}

\author{
Zhengkun Zhang, ${ }^{1}$ Xinyao Xia, ${ }^{2}$ Qian Du, ${ }^{1}$ Lei Xia, ${ }^{1}$ Xiaoyu Ma, ${ }^{2}$ Qiyun $\mathrm{Li}^{1},{ }^{1, \dagger}$ and Wende Liu ${ }^{2, \dagger}$ \\ ${ }^{1}$ Jilin Academy of Agricultural Sciences, Jilin Key Laboratory of Agricultural Microbiology; Key \\ Laboratory of Integrated Pest Management on Crops in Northeast, Ministry of Agriculture, \\ Changchun, Jilin Province 130033, China \\ ${ }^{2}$ State Key Laboratory for Biology of Plant Diseases and Insect Pests, Institute of Plant Protection, \\ Chinese Academy of Agricultural Science, Beijing 100193, China
}

\section{Abstract}

Rhizoctonia solani is an important soil-borne fungal pathogen that causes serious diseases on many agricultural crops and vegetables. Here, we report a complete genome assembly of $R$. solani AG4 (assembly: $45.47 \mathrm{Mb}$; contig $\mathrm{N}_{50}$ : $1.56 \mathrm{Mb}$ ), using a combination of Illumina paired-end and PacBio long-read sequencing data. A total of 267 noncoding RNAs and 11,592 genes were predicted, including 109 genes associated with carbohydrate-active enzymes and 2,488 genes involved in host-pathogen interactions. The complete genome of $R$. solani AG4 represents a valuable base for studying interactions between host plants and pathogenic fungi and to search for potential antimicrobial targets.

\section{Genome Announcement}

Rhizoctonia solani is a significant fungal pathogen that demonstrates a broad host range and causes disease on diverse cereal, vegetable, and forest tree plants, including rice, maize, potato, pepper, bean, cotton, sugar beet, lettuce, melon, and ornamental plants, resulting in huge economic losses (Carling et al. 2002; Ghosh et al. 2018; Rao et al. 2019; Zheng et al. 2013). R. solani is particularly difficult to control because it survives in soil and fields by forming sclerotia, which are condensed bodies of fungal hyphae that can survive in the soil for as long as 2 years (Long et al. 2019). R. solani comprises a species complex of 14 genetic groups called anastomosis groups (AGs), named AG-1 to AG-13 and AG-BI (Carling et al. 2002; Ghosh et al. 2018; González Garcia et al. 2006). R. solani AG-4 has a wide host range, infecting crops in the Chenopodiaceae, Fabaceae, Solanaceae, and Cucurbitaceae families (Yang and Wu 2013). In the present study, we sequenced strain Rhs4ca of $R$. solani AG4 to provide a preliminary understanding of its genetic information.

Previously, 11 assemblies of $R$. solani, including $R$. solani AG1 (Zheng et al. 2013), $R$. solani AG3 (Cubeta et al. 2014), and $R$. solani AG8 (Hane et al. 2014), have been published. All the published $R$. solani genomes were sequenced using the Illumina platform, which produces short reads of less than $150 \mathrm{bp}$. Third-generation sequencing technologies, represented by Oxford Nanopore and Pacific Biosciences (PacBio) single-molecule real-time sequencing

†Corresponding authors: Q. Li; qyli1225@126.com and W. Liu; wendeliu@126.com

Zhengkun Zhang and Xinyao Xia are co-lead authors.

The author(s) declare no conflict of interest.

Accepted for publication 28 February 2021.

\section{Funding}

This work was funded by National Key R\&D Programs grant number 2017 YFD200608.

\section{Keywords}

genome, PacBio, Rhizoctonia solani AG4 


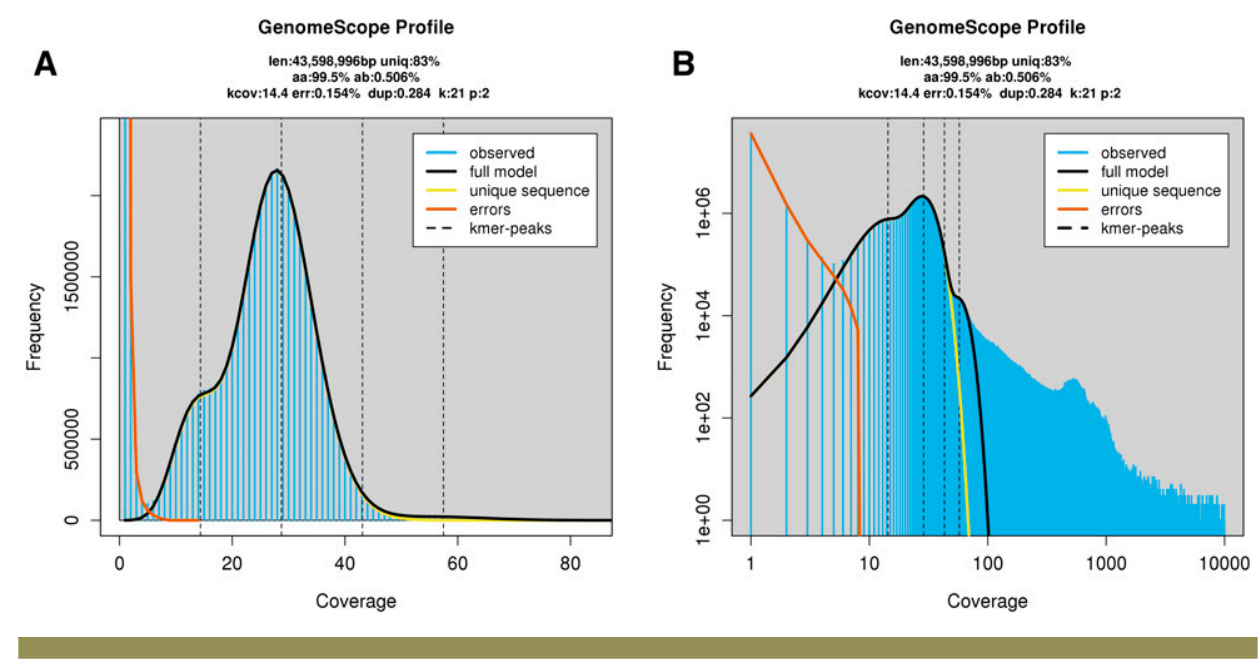

Fig. 1. K-mer analysis of the Rhizoctonia solani Rhs4ca genome. A, The 21-mer coverage distribution of the Illumina paired-end reads. A total of 1,311,292,933 21-k-mers were counted, with a peak depth of 28. B, Partial schematic of the 21-mer coverage distribution. The blue area is the actual observed k-mer distribution. The red line represents k-mers with low frequency, which were considered as sequencing errors. The black lines represent the reliable k-mer data that were used to evaluate the size of the genome. The vertical dashed lines are the k-mer peaks. The yellow line at the bottom represents the size of the repeat region.

technology, offer an effective approach to assemble genomes with high heterozygosity and containing high levels of repetitive sequences (Kyriakidou et al. 2020). For disease-causing fungi, high-quality genome assemblies are crucial because disease-causing genes are often identified among the highly repetitive regions of the genome (Thomma et al. 2016). Considering the high random error rate of PacBio sequencing technology, assembly combining PacBio long reads and error correction using Illumina short reads is a widely used approach to obtain contiguous genomes with a low error rate.

$R$. solani Rhs4ca was isolated from diseased pepper leaves showing root rot symptoms that were collected from the field of the Economic Plant Research Institute, Jilin Academy of Agricultural Sciences, Gongzhuling, Jilin, China. Pure cultures of isolates were obtained using the hyphal tip technique described by Dhingra and Sinclair (1985). Observation of morphological traits, including mycelium branched at right angles with a septum near the branch and a slight constriction at the branch base, was consistent with the description by Yang and Wu (2013). DNA was extracted from mycelia using a REDExtract-N-Amp plant PCR kit (Roche, Basel, Switzerland). The internal transcribed spacer (ITS)-ribosomal DNA region was amplified using the ITS1F and ITS4 PCR primers following the protocol of Weir et al. (2012), and the amplicon was sequenced on an ABI 3500xI Sanger sequencer (Applied Biosystems, Foster City, CA, U.S.A.). The ITS1 and ITS4 sequences were compared with entries in the GenBank database and were found to be most similar to group AG-4 HG-II at 99.27 and $100 \%$ identity, respectively.

The isolated DNA of $R$. solani was sequenced by Beijing Novogene Technology Co., Ltd. (Beijing), using the PacBio Sequel platform (Menlo Park, CA, U.S.A.) and the Illumina HiSeq2500 platform (San Diego, CA, U.S.A.) according to manufacturer protocol. Finally, $7.68 \mathrm{~Gb}$ of long-read data (approximately $169 \times$ coverage) was obtained. The Illumina 350-bp library was PE150 $(2 \times 150$ bp paired-end reads) sequenced and produced $1.51 \mathrm{~Gb}$ of short reads (33x coverage). Based on the lllumina paired-end reads, Jellyfish v2.2.10 (Marçais and Kingsford 2011) and Genomescope v2.0 (Ranallo-Benavidez et al. 2020) were used to perform a genome survey of $R$. solani. As shown in Figure $1 \mathrm{~A}$ and $\mathrm{B}, R$. solani Rhs $4 \mathrm{ca}$ had an estimated size of $43.60 \mathrm{Mb}$ with $0.51 \%$ heterozygosity and $17.04 \%$ repeats. De novo assemble was performed using SMARTdenovo v1.0 from the pure PacBio reads (Liu et al. 2020). To improve the assembly, three rounds of corrections using Racon v1.4.10 (Vaser et al. 2017) with the PacBio data and one round of correction using Pilon v1.23 (Walker et al. 2014) with the lllumina data were completed based on the draft genome. The draft genome was $45.47 \mathrm{Mb}$, comprising 82 contigs and a $48 \% \mathrm{GC}$ content. The $\mathrm{N}_{50}$ of the genome (the sequence length of the shortest contig at $50 \%$ of the total genome length) was $1.56 \mathrm{Mb}$. The

Vol. 34, No. 7, 2021 / 827 
Table 1. Summary statistics of the Rhizoctonia solani genome

\begin{tabular}{lllll} 
Parameters $^{\text {a }}$ & R. solani AG4 & R. solani AG1 & R. solani AG3 & R. solani AG8d \\
Isolate name & Rhs4ca & IA & Rhs1AP & WAC10335 \\
Sequencing technology & PacBio, Illumina & Illumina & Illumina & Illumina \\
Number of contigs & 82 & 6,452 & 6,040 & 7,606 \\
Assembly length (bp) & $45,474,565$ & $36,938,120$ & $51,705,945$ & $39,822,884$ \\
$\mathrm{~N}_{50}$ (bp) & $1,560,204$ & 20,319 & 25,869 & 7,182 \\
GC content (\%) & 48.0 & 47.3 & 48.4 & 48.4 \\
Number of predicted genes & 11,592 & 10,593 & 12,738 & 13,952 \\
BUSCO completeness (\%) & 92.0 & - & - & - \\
Repeat rate (\%) & 19.79 & - & - & - \\
NR database & 11,616 & - & - & - \\
Swiss Prot database & 6,230 & - & - & - \\
CAZYme database & 109 & - & - & - \\
\hline
\end{tabular}

a BUSCO = benchmarking universal single-copy orthologs, $\mathrm{N}_{50}=$ the sequence length of the shortest contig at $50 \%$ of the total genome length, NR = nonredundant, and CAZyme = carbohydrateactive enzyme.

${ }^{\mathrm{b}}$ Zheng et al. 2013.

${ }^{\mathrm{c}}$ Cubeta et al. 2014.

d Hane et al. 2014.

length of the 82 contigs ranged from $22.68 \mathrm{~Kb}$ to $3.44 \mathrm{Mb}$. The final genome was assessed using BUSCO v4.1.4 (Simão et al. 2015), using the lineage-specific profile library fungaI_odb10 with 758 markers, which suggested that $92.0 \%$ of the fungal genes are present in R. solani, $89.8 \%$ of which are complete and single-copy. RepeatModeler v1.0.8 (Smit and Hubley 2008) and RepeatMasker v4.0.9 (Tarailo-Graovac and Chen 2009) were used to identify and mask the repeats, resulting in $9.00 \mathrm{Mb}(19.79 \%)$ of interspersed repeats. Gene prediction was performed using three different $a b$ initio gene annotation programs, namely, Augustus v3.3.3 (Stanke et al. 2006), GeneMark-ES v3.0.4 (Borodovsky et al. 2003), and SNAP v2013.11.29 (Johnson et al. 2008). The gene model of Fusarium graminearum was used to train Augustus and SNAP. Evidence Modeler v1.1.1 (Haas et al. 2008) was used to integrate the consensus gene structures. Finally, a total of 11,859 protein-coding genes of $R$. solani Rhs4ca were predicted. Gene annotation based on the best alignment (E-value < $10^{-5}, k=1$ ) match by BLAST searching in the nonredundant protein (NR) database and Swiss Prot database (Bairoch and Apweiler 2000) was performed using Diamond v0.8.22 (Buchfink et al. 2015). Among the annotated genes, 6,230 (52.53\%) were mapped to Swiss Prot and 11,616 (97.95\%) of their encoded proteins had similarity to proteins in the NR database. A total of 109 genes were mapped to the CAZyme database (Huang et al. 2018) and encoded carbohydrate-active enzymes; 1,531 genes were related to transport, as confirmed by analysis at the Transporter Classification Database (Saier et al. 2006); and 2,488 genes were involved in interactions between the pathogen and the host, as evidenced by analysis at the Pathogen-Host Interactions database (Winnenburg et al. 2006). Infernal v1.1 (Nawrocki and Eddy 2013) and tRNAscan-SE v2.0.6 (Lowe and Eddy 1997) were used to identify 267 noncoding RNAs, including 197 transfer RNAs and 70 ribosomal RNAs. Nine secondary metabolite biosynthesis gene clusters were identified, based on analysis using the antiSMASH fungal v4.0.0 server (Blin et al. 2017) with strict parameters, including two terpene and one nonribosomal peptide synthetase clusters.

Here, the recently published sequences of three $R$. solani isolates from three anastomosis groups were compared with that of strain Rhs4ca (Table 1). The comparison showed that the parameters of assembly length, GC (\%), and the number of predicted genes were consistent among the four sequenced strains. In addition, for the number of contigs parameter, our result of 82 contigs was markedly lower than that of the other three isolates, which were $6,452,6,040$, and 7,606 , respectively. The $\mathrm{N}_{50}$ value was $1.56 \mathrm{Mb}$ for isolate Rhs4ca, indicating a marked improvement in the integrity and continuity of the $R$. solani genome.

In summary, we reported the draft genome of Rhizoctonia solani AG4, a fungal pathogen that causes stem and root rot of many crops and vegetables. A total of 11,592 genes were predicted, including 109 genes related to carbohydrate-active enzymes, 1,531 genes 
related to transport, and 2,488 genes involved in host-pathogen interactions. This draft genome sequence will provide a valuable basis for future research into the pathogenesis of $R$. solani. The genome assembly has been deposited at the National Center for Biotechnology Information under accession number PRJNA673572.

\section{Literature Cited}

Bairoch, A., and Apweiler, R. 2000. The SWISS-PROT protein sequence database and its supplement TrEMBL in 2000. Nucleic Acids Res. 28:45-48.

Blin, K., Wolf, T., Chevrette, M. G., Lu, X., Schwalen, C. J., Kautsar, S. A., Suarez Duran, H. G., de Los Santos, E. L. C., Kim, H. U., Nave, M., Dickschat, J. S., Mitchell, D. A., Shelest, E., Breitling, R., Takano, E., Lee, S. Y., Weber, T., and Medema, M. H. 2017. antiSMASH 4.0-improvements in chemistry prediction and gene cluster boundary identification. Nucleic Acids Res. 45 (W1):W36-W41.

Borodovsky, M., Mills, R., Besemer, J., and Lomsadze, A. 2003. Prokaryotic gene prediction using GeneMark and GeneMark.hmm. Curr. Protoc. Bioinformat. 1:4.5.1-4.5.16.

Buchfink, B., Xie, C., and Huson, D. H. 2015. Fast and sensitive protein alignment using DIAMOND. Nat. Methods 12:59-60.

Carling, D. E., Kuninaga, S., and Brainard, K. A. 2002. Hyphal anastomosis reactions, rDNA-internal transcribed spacer sequences, and virulence levels among subsets of Rhizoctonia solani anastomosis group-2 (AG-2) and AG-BI. Phytopathology 92:43-50.

Cubeta, M. A., Thomas, E., Dean, R. A., Jabaji, S., Neate, S. M., Tavantzis, S., Toda, T., Vilgalys, R., Bharathan, N., Fedorova-Abrams, N., Pakala, S. B., Pakala, S. M., Zafar, N., Joardar, V., Losada, L., and Nierman, W. C. 2014. Draft genome sequence of the plant-pathogenic soil fungus Rhizoctonia solani anastomosis group 3 strain Rhs1AP. Genome Announc. 2:e01072-14.

Dhingra, O. D., and Sinclair, J. B. 1985. Basic plant pathology methods. CRC Press, Inc.. Boca Raton, FL. U.S.A.

Ghosh, S., Kanwar, P., and Jha, G. 2018. Identification of candidate pathogenicity determinants of Rhizoctonia solani AG1-IA, which causes sheath blight disease in rice. Curr. Genet. 64:729-740.

González Garcia, V., Portal, O., and Rubio, S. 2006. Biology and systematics of the form genus Rhizoctonia. Span. J. Agric. Res. 4:55-79.

Haas, B. J., Salzberg, S. L., Zhu, W., Pertea, M., Allen, J. E., Orvis, J., White, O., Buell, C. R., and Wortman, J. R. 2008. Automated eukaryotic gene structure annotation using EVidenceModeler and the Program to Assemble Spliced Alignments. Genome Biol. 9:R7.

Hane, J. K., Anderson, J. P., Williams, A. H., Sperschneider, J., and Singh, K. B. 2014. Genome sequencing and comparative genomics of the broad host-range pathogen Rhizoctonia solani AG8. PLoS Genet. 10:e1004281.

Huang, L., Zhang, H., Wu, P., Entwistle, S., Li, X., Yohe, T., Yi, H., Yang, Z., and Yin, Y. 2018. dbCAN-seq: A database of carbohydrate-active enzyme (CAZyme) sequence and annotation. Nucleic Acids Res. 46:D516-D521.

Johnson, A. D., Handsaker, R. E., Pulit, S. L., Nizzari, M. M., O’Donnell, C. J., and de Bakker, P. I. 2008. SNAP: A web-based tool for identification and annotation of proxy SNPs using HapMap. Bioinformatics 24:2938-2939.

Kyriakidou, M., Anglin, N. L., Ellis, D., Tai, H. H., and Strömvik, M. V. 2020. Genome assembly of six polyploid potato genomes. Sci. Data 7:88.

Liu, H., Wu, S., Li, A., and Ruan, J. 2020. SMARTdenovo: A de novo assembler using long noisy reads. Published online.

Long, J. J., Luna, E. K., Jackson, M., Wheat, W., Jahn, C. E., and Leach, J. E. 2019. Interactions of free-living amoebae with the rice fungal pathogen, Rhizoctonia solani. BMC Res. Notes 12:746.

Lowe, T. M., and Eddy, S. R. 1997. tRNAscan-SE: A program for improved detection of transfer RNA genes in genomic sequence. Nucleic Acids Res. 25:955-964.
Marçais, G., and Kingsford, C. 2011. A fast, lock-free approach for efficient parallel counting of occurrences of k-mers. Bioinformatics 27:764-770.

Nawrocki, E. P., and Eddy, S. R. 2013. Infernal 1.1: 100-fold faster RNA homology searches. Bioinformatics 29:2933-2935.

Ranallo-Benavidez, T. R., Jaron, K. S., and Schatz, M. C. 2020. GenomeScope 2.0 and Smudgeplot for reference-free profiling of polyploid genomes. Nat. Commun. 11:1432.

Rao, T. B., Chopperla, R., Methre, R., Punniakotti, E., Venkatesh, V., Sailaja, B., Reddy, M. R., Yugander, A., Laha, G. S., Madhav, M. S., Sundaram, R. M., Ladhalakshmi, D., Balachandran, S. M., and Mangrauthia, S. K. 2019. Pectin induced transcriptome of a Rhizoctonia solani strain causing sheath blight disease in rice reveals insights on key genes and RNAi machinery for development of pathogen derived resistance. Plant Mol. Biol. 100:59-71.

Saier, M. H., Jr., Tran, C. V., and Barabote, R. D. 2006. TCDB: The transporter classification database for membrane transport protein analyses and information. Nucleic Acids Res. 34:D181-D186.

Simão, F. A., Waterhouse, R. M., loannidis, P., Kriventseva, E. V., and Zdobnov, E. M. 2015. BUSCO: Assessing genome assembly and annotation completeness with single-copy orthologs. Bioinformatics 31:3210-3212.

Smit, A. F., and Hubley, R. 2008. RepeatModeler Open-1.0. Institute for Systems Biology. Published online. http://www.repeatmasker.org.

Stanke, M., Keller, O., Gunduz, I., Hayes, A., Waack, S., and Morgenstern, B. 2006. AUGUSTUS: Ab initio prediction of alternative transcripts. Nucleic Acids Res. 34:W435-W439.

Tarailo-Graovac, M., and Chen, N. 2009. Using RepeatMasker to identify repetitive elements in genomic sequences. Curr. Protoc. Bioinformat. 25:4.10.1114.10.14.

Thomma, B. P. H. J., Seidl, M. F., Shi-Kunne, X., Cook, D. E., Bolton, M. D., van Kan, J. A. L., and Faino, L. 2016. Mind the gap; seven reasons to close fragmented genome assemblies. Fungal Genet. Biol. 90:24-30.

Vaser, R., Sović, I., Nagarajan, N., and Šikić, M. 2017. Fast and accurate de novo genome assembly from long uncorrected reads. Genome Res. 27:737746.

Walker, B. J., Abeel, T., Shea, T., Priest, M., Abouelliel, A., Sakthikumar, S., Cuomo, C. A., Zeng, Q., Wortman, J., Young, S. K., and Earl, A. M. 2014. Pilon: An integrated tool for comprehensive microbial variant detection and genome assembly improvement. PLoS One 9:e112963.

Weir, B. S., Johnston, P. R., and Damm, U. 2012. The Colletotrichum gloeosporioides species complex. Stud. Mycol. 73:115-180.

Winnenburg, R., Baldwin, T. K., Urban, M., Rawlings, C., Köhler, J., and Hammond-Kosack, K. E. 2006. PHI-base: A new database for pathogen host interactions. Nucleic Acids Res. 34:D459-D464.

Yang, Y. G., and Wu, X. H. 2013. First report of potato stem canker caused by Rhizoctonia solani AG4 HGll in Gansu Province, China. Plant Dis. 97:840.

Zheng, A., Lin, R., Zhang, D., Qin, P., Xu, L., Ai, P., Ding, L., Wang, Y., Chen, Y., Liu, Y., Sun, Z., Feng, H., Liang, X., Fu, R., Tang, C., Li, Q., Zhang, J., Xie, Z., Deng, Q., Li, S., Wang, S., Zhu, J., Wang, L., Liu, H., and Li, P. 2013. The evolution and pathogenic mechanisms of the rice sheath blight pathogen. Nat. Commun. 4:1424. 